\title{
A minimal set of internal control genes for gene expression studies in head and neck squamous cell carcinoma
}

\author{
Vinayak Palve $^{1}$, Manisha Pareek ${ }^{1}$, Neeraja M Krishnan ${ }^{1}$, Gangotri Siddappa ${ }^{2}$, Amritha Suresh ${ }^{2}$, Moni A \\ Kuriakose $^{2}$, Binay Panda ${ }^{\text {Corresp. }} 1$ \\ ${ }^{1}$ Ganit Labs, Bio-IT Centre, Institute of Bioinformatics and Applied Biotechnology, Bangalore, India \\ 2 Head and Neck Oncology, Mazumdar Shaw Centre for Translational Research, Bangalore, India \\ Corresponding Author: Binay Panda \\ Email address: binay@ganitlabs.in
}

Selection of the right reference gene(s) is crucial in the analysis and interpretation of gene expression data. The aim of the present study was to discover and validate a minimal set of internal control genes in head and neck tumor studies. We analyzed data from multiple sources (in house whole-genome gene expression microarrays, previously published qPCR data and RNA-seq data from TCGA) to come up with a list of 18 genes (discovery set) that had lowest variance and high level of expression across tumors and their matched normal samples. The genes in the discovery set were ranked using four different algorithms (BestKeeper, geNorm, NormFinder, and comparative delta $\mathrm{Ct}$ ) and a web-based comparative tool, RefFinder, for their stability and variance in expression across tissues. Finally, we validated their expression using qPCR in an additional set of tumor:matched normal samples that resulted in five genes (RPL30, RPL27, PSMC5, MTCH1 and OAZ1), out of which RPL30 and RPL27 were most stable and were abundantly expressed across the tissues. Our data suggest that RPL30 or RPL27 in combination with either PSMC5 or MTCH1 or OAZ1 can be used as a minimal set of control genes in head and neck tumor gene expression studies. 
1 A minimal set of internal control genes for gene expression studies in head and

2 neck squamous cell carcinoma.

3 Vinayak Palve ${ }^{1}$, Manisha Pareek ${ }^{1}$, Neeraja M Krishnan 1 , Gangotri Siddappa ${ }^{2}$, Amritha

4 Suresh $^{2}$, Moni A Kuriakose ${ }^{2}$ and Binay Panda ${ }^{1 *}$

5

$6{ }^{1}$ Ganit Labs, Bio-IT Centre, Institute of Bioinformatics and Applied Biotechnology,

7 Bangalore, India

$8{ }^{2}$ Head and Neck Oncology, Mazumdar Shaw Centre for Translational Research,

9 Bangalore, India

10

11

12

* Corresponding author

13

Binay Panda: binay@ganitlabs.in

14

15

16

17 


\section{Abstract}

Selection of the right reference gene(s) is crucial in the analysis and interpretation of gene expression data. The aim of the present study was to discover and validate a minimal set of internal control genes in head and neck tumor studies. We analyzed data from multiple sources (in house whole-genome gene expression microarrays, previously published qPCR data and RNA-seq data from TCGA) to come up with a list of 18 genes (discovery set) that had lowest variance and high level of expression across tumors and their matched normal samples. The genes in the discovery set were ranked using four different algorithms (BestKeeper, geNorm, NormFinder, and comparative delta Ct) and a web-based comparative tool, RefFinder, for their stability and variance in expression across tissues. Finally, we validated their expression using qPCR in an additional set of tumor:matched normal samples that resulted in five genes (RPL30, RPL27, PSMC5, MTCH1 and OAZ1), out of which RPL30 and RPL27 were most stable and were abundantly expressed across the tissues. Our data suggest that RPL30 or RPL27 in combination with either PSMC5 or MTCH1 or OAZ1 can be used as a minimal set of control genes in head and neck tumor gene expression studies. 


\section{Introduction}

Internal control genes or housekeeping genes are important in obtaining reliable and reproducible data from gene expression studies in cancer (Eisenberg \& Levanon 2013). Internal control genes should be abundantly and uniformly expressed across tumor and normal tissues and across different experimental conditions (Janssens et al. 2004). Cancers of head and neck region is the sixth most common cancer worldwide with an incidence of 550,000 cases annually (Ferlay et al. 2010). Past studies argue the lack of uniformity of expression on internal control genes based on experimental conditions (De Jonge et al. 2007; Greer et al. 2010). Although there are previous reports that describe the reference or internal control genes in head and neck squamous cell carcinoma (HNSCC) gene expression studies (Lallemant et al. 2009), use of highthroughput data like microarrays and RNA-seq from tumors and their matched normal tissues following a confirmation of expression in independent set of samples are scant in the literature. Additionally, it is crucial that the expression of internal control genes remains unaltered across temporal, spatial and experimental conditions that takes into account of the genes with a wide dynamic range of expression. Therefore, revisiting the validity of widely used genes like $A C T B, T U B B$ and $G A P D H$ is required along with discovery of a minimal set of internal control genes in HNSCC gene expression studies that use expression data from patient cohorts from different geography (Krishnan et al. 2015; Krishnan et al. 2016) and large consortia like TCGA (Cancer Genome Atlas 2015).

In the present study, we analyzed HNSCC gene expression data from three sources; in house microarray data (Krishnan et al. 2015; Krishnan et al. 2016) (n=21), 
61 TCGA RNA-seq data (Cancer Genome Atlas 2015) (n=42) and qPCR data on individual

62 genes from the previously published studies (Bär et al. 2009; Eisenberg \& Levanon 2013; Lallemant et al. 2009; Martin 2016; Vandesompele et al. 2002); to come up with a set of genes (discovery set) that are stably and robustly expressed with least variance across tumor:normal pairs. We subsequently validated the expression of the discovery set in additional tumor:normal pairs $(n=14)$ using qPCR and obtained a minimal set of two housekeeping genes by comparing and ranking their expression during validation.

\section{Materials and Methods}

Microarray data processing

The gene expression profiling using Illumina HumanHT-12 v4 expression 
RNA-seq data processing

RSEM-processed RNA-Seq gene expression values (TCGA pipeline, Level 3) were downloaded from the old TCGA repository (https://genomecancer.ucsc.edu/proj/site/hgHeatmap/). The transcripts per kilobase million (TPM) values for all the genes were extracted from the Level 3 files. Further, genes that showed zero or NA values in any of these samples were eliminated, and the log fold change values between the respective tumor and normal samples calculated by taking a log transformation of the ratios between expression values for tumor and normal. We filtered expression data that fulfilled three criteria for all samples; expressed at $>=3$ TPM, tumor/normal ratio: $0.9-1.1$ and standard deviation across all the tissues $<0.5$. Finally genes were short-listed from a larger gene list expressed at a high level in both the microarray and the RNA-seq data (Supplementary Table 1) for the discovery set.

Discovery set

In addition to the microarray and RNA-seq gene expression data, the most commonly used internal control genes form the HNSCC gene expression literature (Bär et al. 2009; Eisenberg \& Levanon 2013; Lallemant et al. 2009; Martin 2016; Vandesompele et al. 2002) were also considered to select the discovery set $(n=18)$ (Supplementary Table 2). These genes were used for validation in an independent set of tumor:normal pairs $(n=14)$ using qPCR.

\section{Patient samples}

Patients were accumulated voluntarily after obtaining informed consents from each patient and as per the guidelines from the institutional ethics committee of the 
105 Mazumadar-Shaw Cancer Centre (IRB Approval number: NHH/MEC-CL/2014/197). All

106 the tissues were frozen immediately in liquid nitrogen and stored at $-80^{\circ} \mathrm{C}$ until further

107 use. Only those tumors with squamous cell carcinoma (with at least $70 \%$ tumor cells

108 and with confirmed diagnosis) and their adjacent normal tissues as confirmed by

109 histology (Supplementary Figure 1) were included in the current study. Patients

110 underwent staging according to AJCC criteria, and curative intent treatment as per

111 NCCN guidelines involving surgery with or without post-operative adjuvant radiation or

112 chemo-radiation at the Mazumdar Shaw Cancer Centre were accrued for the validation 113 study.

114 RNA extraction and cDNA synthesis

115 The total RNA was extracted from $25 \mathrm{mg}$ of tissues using the RNeasy mini kit 116 (Qiagen, cat:74104) with on-column digestion of DNA using RNase free DNase set

117 (Qiagen, cat:79254) as per manufacturer's instructions. The RNA quality was checked 118 using gel electrophoresis and Agilent Bioanalyzer. Five hundred nanogram of total RNA 119 was subjected to cDNA synthesis using Takara's Prime Script first strand cDNA 120 synthesis kit (cat: 6110A).

121

Quantitative real-time PCR (qPCR)

122 Quantitative PCR was carried out using KAPA Biosystem's SYBR Fast qPCR 123 universal master mix (cat: KK4601). The primer sequences for all the 18 discovery set 124 reference genes and the amplification conditions are mentioned in Table 1A. The 125 primers were either designed for this study or were chosen from the literature (Campos 126 et al. 2009) or from the online resources (https://primerdepot.nci.nih.gov/ and 
127 https://pga.mgh.harvard.edu/primerbank/). All amplification reactions were carried out in

128 triplicates, using three negative cotrols: no template control (NTC) with nuclease free

129 water (Ambion, cat: AM9932), no amplification control (NAC) and no primer control

130 (NPC) in each amplification plate. We have followed the guidelines for quantitative real-

131 time PCR experiments as suggested previously (Bustin et al. 2010).

132 Statistical analysis

133 For stability comparison of the internal candidate reference genes obtained using 134 the discovery set, we analyzed the validation data independently using four most 135 commonly used algorithms, Genorm (Vandesompele et al. 2002), Normfinder

136 (Andersen et al. 2004), Bestkeeper (Pfaffl et al. 2004) and Comparative Ct (Schmittgen

137 \& Livak 2008). In addition to the comparative Ct method, we also calculated the values

138 of mean standard deviation (Silver et al. 2006) of Ct for each gene. The results from the

139 above tools were compared with an online tool Reffinder (Xie et al. 2012) and the

140 results were interpreted and presented separately. Graphpad prism software version 5

141 was used to analyze the data and plot the graphs.

\section{Results and Discussion}

The schema for selecting genes for discovery set is depicted in Figure 1. After analyzing data from all sources, 18 genes were selected that had least variance across all the tumor and normal samples, were well annotated and had some biology known

147 (Table 1A and Supplementary Table 2). Standard curves reflecting the linear regression $148\left(R^{2}>0.9\right)$ and amplification efficiency (Supplementary Figure 2, Supplementary Table 3) 
149 were generated for the select candidate genes. Primers for all the genes showed 150 specific amplifications as shown by the dissociation curves (Supplementary Figure 3)

151 and by gel electrophoresis of the amplified products (Supplementary Figure 4). Data 152 from qPCR validation of genes (Figure 2A, Supplementary Table 3) showed variable 153 levels of expression for the 18 genes in different validation samples. As Figure 2B 154 indicates independent results from the four different algorithms (Genorm, Normfinder, 155 Bestkeeper and Comparative $\mathrm{Ct}$ ) and the online tool RefFinder. As the tool RefFinder 156 does not take amplification efficiency into account and merely sums up results from 157 other tools, we did not rely on the results from RefFinder but used it as one of the tools 158 along with the other four to interpret the results independently. Out of the 18 genes 159 across all the samples, we ranked the expression of top 11 stably expressed genes 160 namely, RPL30, RPL27, PSMC5, OAZ1, MTCH1, TSPAN21, DARS, MKRN1, RPS13, 161 RPL5 and RPL37A. Although, there was some inconsistency between the top-ranked 162 genes among the different algorithms/tools, for the genes RPL27 and RPL30 the data

163 164 was consistent across all the algorithms As both of RPL27 and RPL30 belong to the same ribosomal family proteins, and in order to avoid any potential error due to mutual expression alterations of those genes, we considered inclusion of the next three genes PSMC5, MTCH1 and OAZ1, the expression of which were stable across all the samples. Therefore, RPL27 or RPL30 in combination with any one of the three (PSMC5 / MTCH1 / OAZ1) fulfilled all the 3 criteria for ideal internal control genes, least variance across samples, high-level of expression in both tumors and normal and at the top of the rank by the algorithms tested. 
171 The aim of the current study was not to select a set of genes for a particular tumor stage

172 and/or differentiation and in response to drug treatment but to select genes that are

173 robustly expressed across multiple tumor stages and differentiation and across tumor

174 and their matched normal samples. In HNSCC, previous attempts have been made to

175 come up with a set of internal control genes using qPCR data (Lallemant et al. 2009;

176 Martin 2016) or using tumors and healthy controls (Reddy et al. 2016). However, it is

177 important that the discovery is made from a transcriptome-wide study that takes into

178 account data from tumors and their matched control samples. This is especially

179 important as we have data on HNSCC from large consortia, like TCGA (Cancer

180 Genome Atlas 2015), and data from HNSCC from non-TCGA cohorts (Krishnan et al.

181 2015; Krishnan et al. 2016). It is not surprising that the genes in our discovery set code

182 for structural proteins (ribosomal, cell structure and integrity) and perform essential

183 functions like glycolysis and translation (Supplementary Table 2). In the validation study,

184 RPL30, RPL27, PSMC5, OAZ1 and MTCH1 came as the top 5 ranked genes with

185 stable expression across all the samples. Both RPL27 and RPL30 were previously

186 found to be one the most stable candidate internal control genes (De Jonge et al. 2007;

187 Lallemant et al. 2009) with stable expression in salivary samples of oral cancer patients

188 (Martin 2016). Additionally, previous findings on non-HNSCC tumors had RPL27,

189 RPL30 and MTCH1 in the list of top internal control genes (Popovici et al. 2009).

190 Although, our data supports PSMC5, OAZ1 and MTCH1 were stably expressed and

191 ranked after RPL30 and RPL27 by RefFinder, they were expressed at a lower level than

192 RPL30 and RPL27. Therefore, we suggest using RPL30 or RPL27 in combination with 
193 one of the three genes (PSMC5, MTCH1, OAZ1) to serve as a minimal set of control

194 genes in head and neck tumor gene expression studies.

195

196

Our study suffers from some shortcomings. First, our sample size was small.

197

198

199

200

201

202

203

204

205

206

207

208

209

210

211

212

213

214

215

Second, the GPCR studies, although highly sensitive and widely used, could have resulted in a bias in our gene expression data. RNA input volume, cDNA synthesis efficiency, pipetting volumes and accuracy, and primer amplification efficiencies are some of the factors that may affect qPCR results (Bustin et al. 2009). Other factors that might have influenced our selection of the discovery set are inter-platform and -assay variability, temporal, spatial and experimental conditions (for example, drug treatment), and presence of transcript variants in tumor samples. A larger sample size for different experimental conditions and data from cell lines assayed by using a single kit and a single platform (for example, total transcriptome RNA-seq using a single provider's platform and assay version) may provide answers to these in the future. Third, the selection of genes in our study might have got biased as a result of the field effect (due to use of adjacent normal tissues). Use of true normal tissues may circumvent this issue. However, due to the stringency of the ethics committee rules to obtain additional normal tissues from patients without a clinical reason(s), studies with true normal tissues, outside of the adjacent normal, are least likely to take place. Fourth, in our study, like previously reported [24], a difference in the ranking of genes may be toolspecific and certain parameters (like QPCR amplification efficiency) might have influenced the ranking of the genes. A better design and a robust statistical tool to rank genes may overcome this limitation in the future. 


\section{Conclusions}

Although past studies are heavily reliant on the use of $A C T B, T U B B$ and GAPDH to normalize expression data, evidence suggests that the expression of these genes vary greatly and are not suitable internal control genes (Glare et al. 2002; Oliveira et al.

222 control genes may vary based on experimental conditions and tumor type. Therefore, 223 we used a larger discovery set to validate internal control genes across HNSCC tumors 224 and their paired normal samples to come up with a set of robust and stably expressed 225 genes (RPL30, RPL27, PSMC5, MTCH1 and OAZ1) across tissues.

\section{List of abbreviations}

HNSCC: Head and neck squamous cell carcinoma

RPL30: 60S ribosomal protein L30;

RPL27: 60S ribosomal protein L27

231 NTC: No template control

232 NAC: No amplification control

233 NPC: No primer control

\section{Acknowledgement}

235 The authors thank Janani Hariharan in helping with the RNA-seq data analyses. 


\section{References}

Andersen CL, Jensen JL, and Ørntoft TF. 2004. Normalization of real-time quantitative reverse transcription-PCR data: a model-based variance estimation approach to identify genes suited for normalization, applied to bladder and colon cancer data sets. Cancer research 64:5245-5250.

Bär M, Bär D, and Lehmann B. 2009. Selection and validation of candidate housekeeping genes for studies of human keratinocytes-review and recommendations. Journal of Investigative Dermatology 129:535-537.

Bustin SA, Beaulieu JF, Huggett J, Jaggi R, Kibenge FS, Olsvik PA, Penning LC, and Toegel S. 2010. MIQE precis: Practical implementation of minimum standard guidelines for fluorescence-based quantitative real-time PCR experiments. BMC Mol Biol 11:74.

Bustin SA, Benes V, Garson JA, Hellemans J, Huggett J, Kubista M, Mueller R, Nolan T, Pfaffl MW, and Shipley GL. 2009. The MIQE guidelines: minimum information for publication of quantitative real-time PCR experiments. Clinical chemistry 55:611-622.

Campos MS, Rodini CO, Pinto-Junior DS, and Nunes FD. 2009. GAPD and tubulin are suitable internal controls for qPCR analysis of oral squamous cell carcinoma cell lines. Oral Oncol 45:121-126.

Cancer Genome Atlas N. 2015. Comprehensive genomic characterization of head and neck squamous cell carcinomas. Nature 517:576-582.

De Jonge HJ, Fehrmann RS, de Bont ES, Hofstra RM, Gerbens F, Kamps WA, de Vries EG, van der Zee AG, te Meerman GJ, and ter Elst A. 2007. Evidence based selection of housekeeping genes. PLoS One 2:e898.

Du P, Kibbe WA, and Lin SM. 2008. lumi: a pipeline for processing Illumina microarray. Bioinformatics 24:1547-1548.

Eisenberg E, and Levanon EY. 2013. Human housekeeping genes, revisited. Trends in Genetics 29:569-574.

Ferlay J, Shin HR, Bray F, Forman D, Mathers C, and Parkin DM. 2010. Estimates of worldwide burden of cancer in 2008: GLOBOCAN 2008. Int J Cancer 127:2893-2917.

Glare E, Divjak M, Bailey M, and Walters E. 2002. $\beta$-Actin and GAPDH housekeeping gene expression in asthmatic airways is variable and not suitable for normalising mRNA levels. Thorax 57:765-770.

Greer S, Honeywell R, Geletu M, Arulanandam R, and Raptis L. 2010. Housekeeping genes; expression levels may change with density of cultured cells. Journal of immunological methods 355:76-79.

Janssens N, Janicot M, Perera T, and Bakker A. 2004. Housekeeping genes as internal standards in cancer research. Molecular Diagnosis 8:107-113.

Johnson WE, Li C, and Rabinovic A. 2007. Adjusting batch effects in microarray expression data using empirical Bayes methods. Biostatistics 8:118-127.

Krishnan N, Gupta S, Palve V, Varghese L, Pattnaik S, Jain P, Khyriem C, Hariharan A, Dhas K, Nair J, Pareek M, Prasad V, Siddappa G, Suresh A, Kekatpure V, Kuriakose M, and Panda B. 2015. Integrated analysis of oral tongue squamous cell carcinoma identifies key variants and pathways linked to risk habits, HPV, clinical parameters and tumor recurrence. F1000Res 4:1215.

Krishnan NM, Dhas K, Nair J, Palve V, Bagwan J, Siddappa G, Suresh A, Kekatpure VD, Kuriakose MA, and Panda B. 2016. A Minimal DNA Methylation Signature in Oral 
Tongue Squamous Cell Carcinoma Links Altered Methylation with Tumor Attributes. Mol Cancer Res 14:805-819.

Lallemant B, Evrard A, Combescure C, Chapuis H, Chambon G, Raynal C, Reynaud C, Sabra $O$, Joubert D, and Hollande F. 2009. Reference gene selection for head and neck squamous cell carcinoma gene expression studies. BMC Molecular Biology 10:78.

Martin JL. 2016. Validation of Reference Genes for Oral Cancer Detection Panels in a Prospective Blinded Cohort. PLoS One 11:e0158462.

Oliveira JG, Prados RZ, Guedes ACM, Ferreira PC, and Kroon EG. 1999. The housekeeping gene glyceraldehyde-3-phosphate dehydrogenase is inappropriate as internal control in comparative studies between skin tissue and cultured skin fibroblasts using Northern blot analysis. Archives of Dermatological Research 291:659-661.

Pfaffl MW, Tichopad A, Prgomet C, and Neuvians TP. 2004. Determination of stable housekeeping genes, differentially regulated target genes and sample integrity: BestKeeper-Excel-based tool using pair-wise correlations. Biotechnology letters 26:509515.

Popovici V, Goldstein DR, Antonov J, Jaggi R, Delorenzi M, and Wirapati P. 2009. Selecting control genes for RT-QPCR using public microarray data. BMC bioinformatics 10:42.

Reddy RB, Bhat AR, James BL, Govindan SV, Mathew R, Ravindra DR, Hedne N, Illiayaraja J, Kekatpure V, Khora SS, Hicks W, Tata P, Kuriakose MA, and Suresh A. 2016. MetaAnalyses of Microarray Datasets Identifies ANO1 and FADD as Prognostic Markers of Head and Neck Cancer. PloS one 11:e0147409.

Schmittgen TD, and Livak KJ. 2008. Analyzing real-time PCR data by the comparative CT method. Nature protocols 3:1101-1108.

Selvey S, Thompson EW, Matthaei K, Lea RA, Irving MG, and Griffiths LR. 2001. $\beta$-Actin—an unsuitable internal control for RT-PCR. Molecular and cellular probes 15:307-311.

Silver, N., Best, S., Jiang, J., \& Thein, S. L. 2006. Selection of housekeeping genes for gene expression studies in human reticulocytes using real-time PCR. BMC molecular biology, 7: 33 .

Vandesompele J, De Preter K, Pattyn F, Poppe B, Van Roy N, De Paepe A, and Speleman F. 2002. Accurate normalization of real-time quantitative RT-PCR data by geometric averaging of multiple internal control genes. Genome biology 3:research0034. 0031.

Xie F, Xiao P, Chen D, Xu L, and Zhang B. 2012. miRDeepFinder: a miRNA analysis tool for deep sequencing of plant small RNAs. Plant molecular biology 80:75-84.

Zhong $\mathrm{H}$, and Simons JW. 1999. Direct comparison of GAPDH, $\beta$-actin, cyclophilin, and $28 \mathrm{~S}$ rRNA as internal standards for quantifying RNA levels under hypoxia. Biochemical and biophysical research communications 259:523-526. 
321 Figure Legends:

322 Figure 1: Schema indicating the selection criteria and validation of the internal control

323 genes.

324 Figure 2: Expression of genes in the discovery set across tumors and normal samples

325 (A). The Ct values were generated from qPCR experiments and plotted as box plots 326 with median values as lines and boxes indicating 10-90 percentile values and the 327 whiskers as the maximum and minimum values. Ranking of 18 genes using different 328 tools (B). Y-axis for different tools represents; stability value (Genorm, Normfinder), 329 Std.dev+-(CP)(Bestkeeper), 2-(delCt) (Comparative Ct method) and geomean of ranking 330 (RefFinder). The columns in light grey represent stable genes across tumors and 331 normal samples.

332

333 Supplementary Figure and Table Legends

334 Supplementary Figure 1: Representative histological images of the adjacent normal 335 tissues.

336 Supplementary Figure 2: Standard curves of 18 candidate housekeeping genes in the 337 discovery set. The qPCR was performed using 3 times dilution of the template. $\mathrm{R}^{2}$ 338 indicates the correlation coefficient and amplification efficiencies on the basis of the 339 slopes.

340 Supplementary Figure 3: Dissociation curves for the 18 candidate housekeeping 341 genes in the discovery set. The single peak of dissociation curve in qPCR performed for 342 all the genes indicates the specific amplification. 
343 Supplementary Figure 4: Electrophoresis of the PCR amplicons loaded on a 2\%

344 agarose gel.

345 Supplementary Table 1: A larger set of genes common between the microarray data

346 and RNA-seq data with high expression in both tumor and normal samples.

347 Supplementary Table 2: Genes selected in the discovery set and their function. 
Figure 1

Schema indicating the selection criteria and validation of the internal control genes.

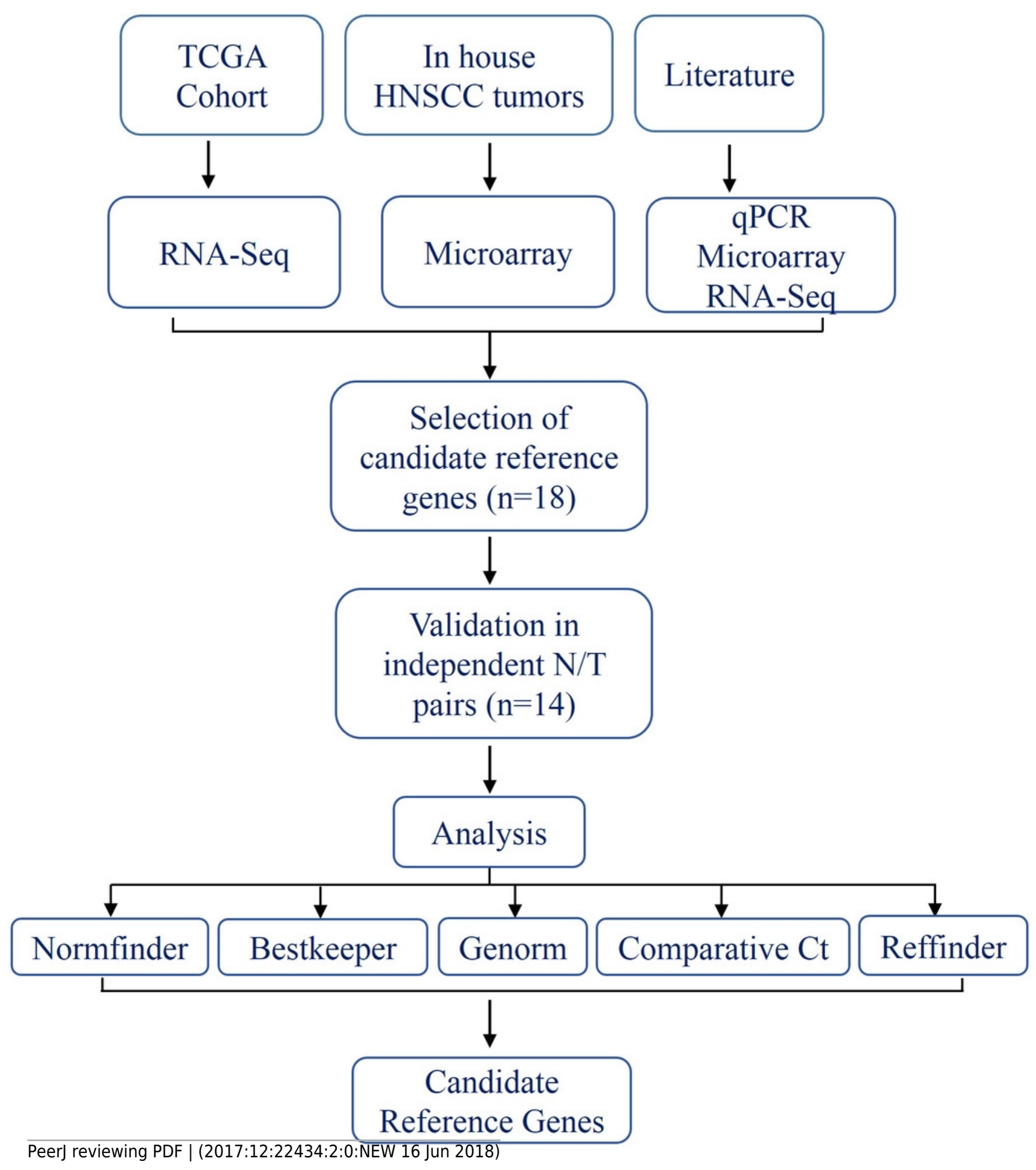




\section{Figure 2}

Expression of genes in the discovery set across tumors and normal samples (A).

The Ctvalues were generated from qPCR experiments and plotted as box plots with median values as lines and boxes indicating 10-90 percentile values and the whiskers as the maximum and minimum values. Ranking of 18 genes using different tools (B). Y-axis for different tools represents; stability value (Genorm, Normfinder), Std.dev+-(CP)(Bestkeeper), $2^{\text {-(delct) }}$ (Comparative Ct method) and geomean of ranking (RefFinder). The columns in light grey represent stable genes across tumors and normal samples. 
$\mathbf{A}$
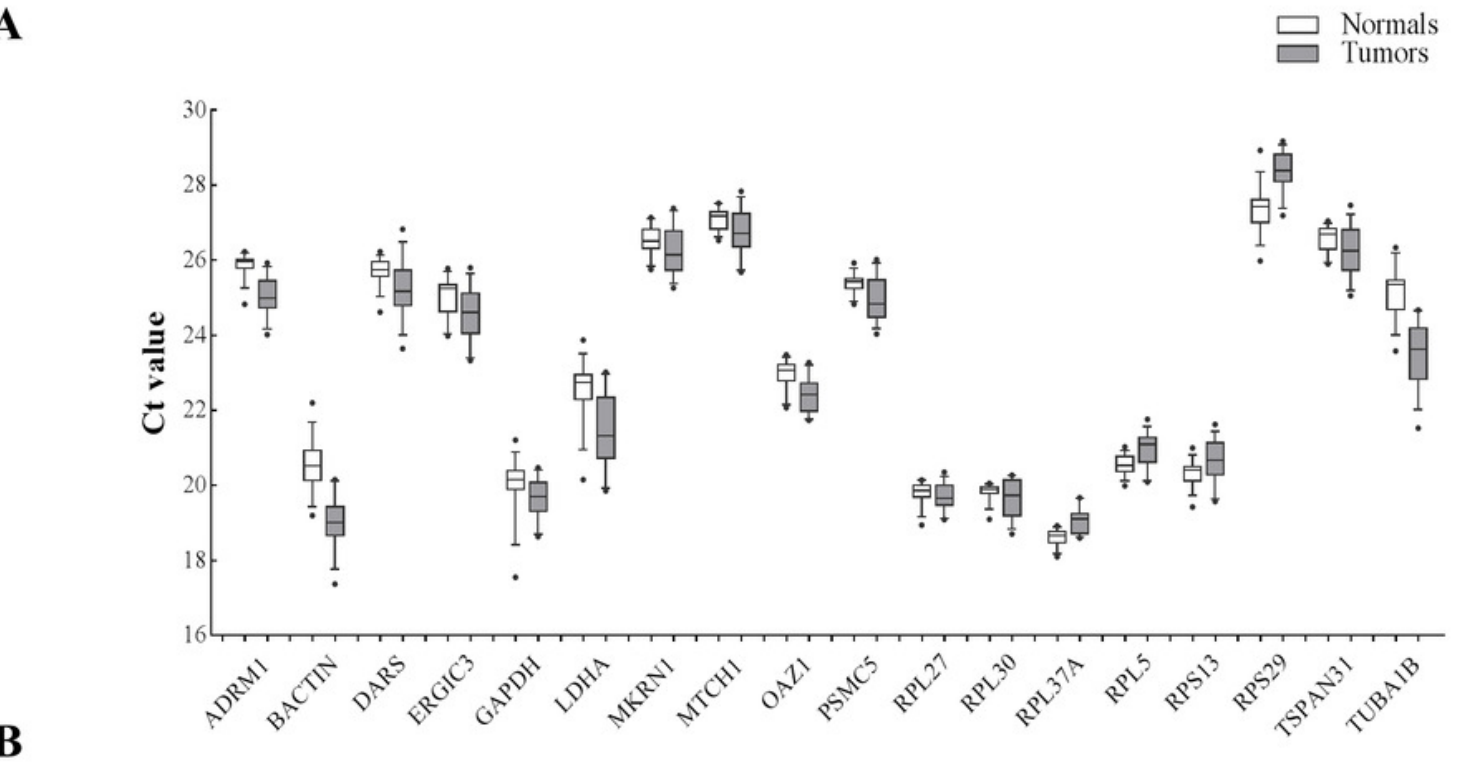

B
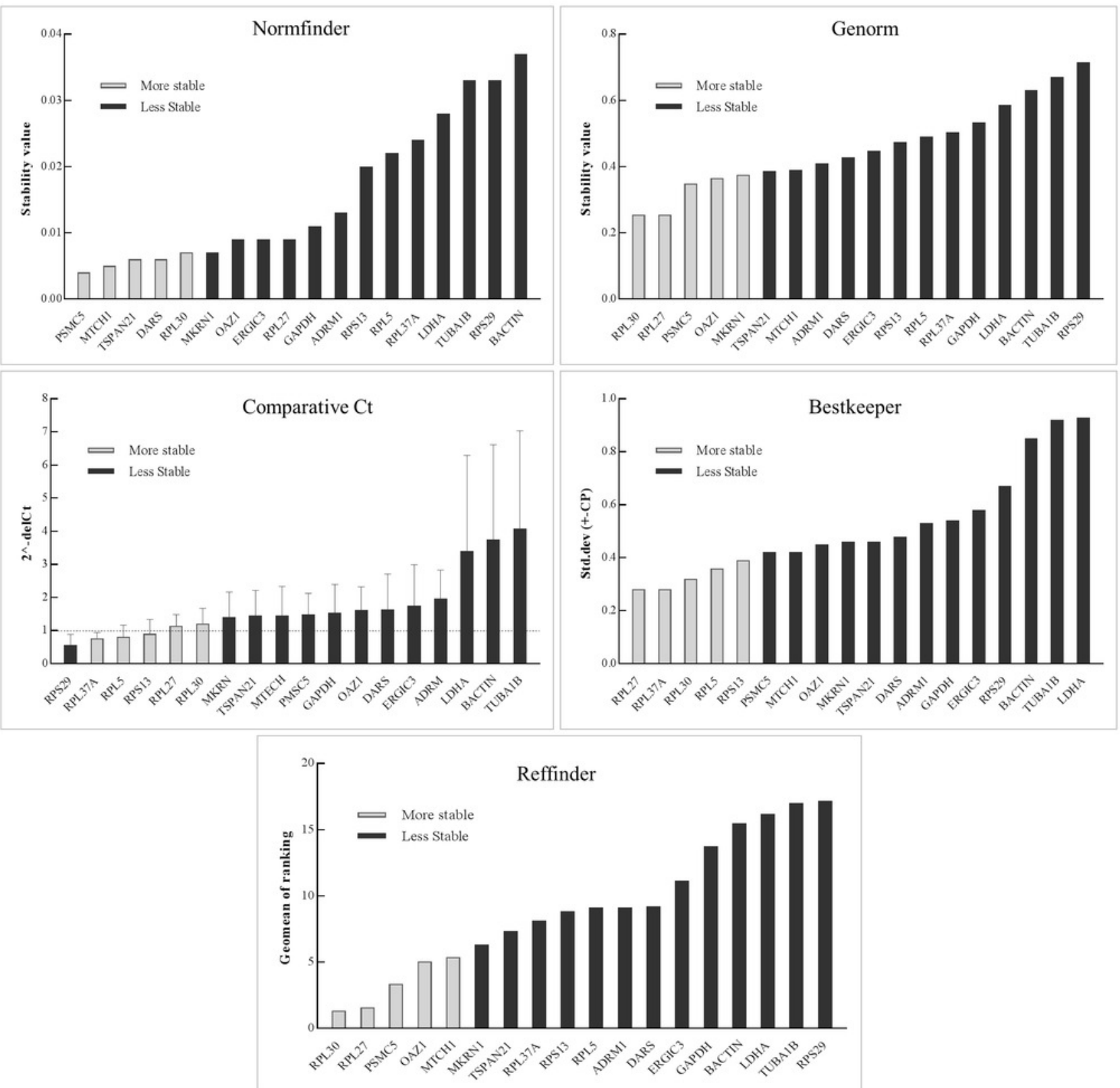


\section{Table $\mathbf{1}$ (on next page)}

Primers used in the current study (A).

The Reference column number indicates- 1: primers designed for the study; 2: primers from Hendrik de Jonge et al., 2007; 3: primers from Primerdepot (https://primerdepot.nci.nih.gov/); 4: primers from primer bank (https://pga.mgh.harvard.edu/primerbank/); 5: primers from Campos et al, 2009. Results from different tools on the internal control genes and their ranks as obtained using the validation samples (B). 


\begin{tabular}{|c|c|c|c|c|c|c|c|c|}
\hline \multicolumn{9}{|l|}{ A } \\
\hline No & Gene & $\mathbf{F} / \mathbf{R}$ & Sequence & $\begin{array}{l}\text { Genomic } \\
\text { Position }\end{array}$ & $\begin{array}{c}\text { Exon- } \\
\text { Intron } \\
\text { Juncti } \\
\text { on } \\
\end{array}$ & $\begin{array}{c}\text { Intron } \\
\text { spanni } \\
\text { ng }\end{array}$ & $\begin{array}{c}\text { Amplicon } \\
\text { Size } \\
\text { (bp) }\end{array}$ & Reference \\
\hline \multirow{2}{*}{1} & \multirow{2}{*}{ RPL27 } & $\mathrm{F}$ & ACAATCACCTAATGCCCACA & \multirow{2}{*}{$\frac{\operatorname{chr17:43000081-}}{\underline{43002884}}$} & \multirow{2}{*}{$\checkmark$} & \multirow{2}{*}{$\sqrt{ }$} & \multirow{2}{*}{146} & \multirow{2}{*}{1} \\
\hline & & $\mathrm{R}$ & GCCTGTCTTGTATCTCTCTTCAA & & & & & \\
\hline \multirow{2}{*}{2} & \multirow{2}{*}{ RPS29 } & $\mathrm{F}$ & GCACTGCTGAGAGCAAGATG & \multirow{2}{*}{$\frac{\operatorname{chr} 14: 49577820-}{\underline{49586363}}$} & \multirow{2}{*}{$\checkmark$} & \multirow{2}{*}{ v } & \multirow{2}{*}{213} & \multirow{2}{*}{$\underline{2}$} \\
\hline & & $\mathrm{R}$ & ATAGGCAGTGCCAAGGAAGA & & & & & \\
\hline \multirow{2}{*}{3} & \multirow{2}{*}{ OAZ1 } & $\mathrm{F}$ & GGATCCTCAATAGCCACTGC & \multirow{2}{*}{$\frac{\operatorname{chr} 19: 2269621-}{\underline{2271410}}$} & \multirow{2}{*}{ - } & \multirow{2}{*}{$\checkmark$} & \multirow{2}{*}{150} & \multirow{2}{*}{$\underline{2}$} \\
\hline & & $\mathrm{R}$ & TACAGCAGTGGAGGGAGACC & & & & & \\
\hline \multirow{2}{*}{4} & \multirow{2}{*}{ RPL30 } & $\mathrm{F}$ & ACAGCATGCGGAAAATACTAC & \multirow{2}{*}{$\frac{\text { chr8:98041745- }}{\underline{98042696}}$} & \multirow{2}{*}{-} & 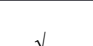 & 158 & 2 \\
\hline & & $\mathrm{R}$ & AAAGGAAAATTTTGCAGGTTT & & & 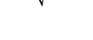 & 100 & $\underline{\underline{a}}$ \\
\hline & & $\mathrm{F}$ & TTGTTGGGGTTGGTGCTGTT & chr11:18396916- & & 1 & & 1 \\
\hline 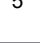 & LUHA & $\mathrm{R}$ & AAGGCTGCCATGTTGGAGAT & 18399514 & - & $v$ & 138 & 1 \\
\hline 6 & $A C T R$ & $\mathrm{~F}$ & CCTTGCACATGCCGGAG & chr7:5529603- & 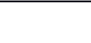 & 1 & 112 & 3 \\
\hline 0 & ACID & $\mathrm{R}$ & GCACAGAGCCTCGCCTT & $\underline{5530574}$ & - & $v$ & 112 & $\underline{\underline{3}}$ \\
\hline 7 & $P P C 12$ & $\mathrm{~F}$ & CGTCCCCACTTGGTTGAAGTT & chr11:17075584- & J & 1 & 120 & 1 \\
\hline$r$ & RPSIS & $\mathrm{R}$ & CGTACTTGTGCAACACCATGT & $\underline{17077438}$ & $v$ & $v$ & 129 & 1 \\
\hline 8 & GAPDH & $\mathrm{F}$ & GCATCCTGGGCTACACTGA & chr12:6537873- & - & $y$ & 162 & 5 \\
\hline 0 & 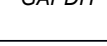 & $\mathrm{R}$ & ССАССАСССТGTTGCTGTA & $\underline{6538138}$ & - & $\checkmark$ & 102 & o \\
\hline 0 & TURM18 & $\mathrm{F}$ & GTCGCCTTCGCCTCCTAATC & chr12:49129610- & 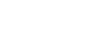 & 1 & 116 & 1 \\
\hline 9 & IUBATB & $\mathrm{R}$ & TCACTTGGCATCTGGCCATC & $\underline{49131330}$ & - & $v$ & 146 & 1 \\
\hline 10 & $P S M C 5$ & $\mathrm{~F}$ & TTGACGGACCAGAGCAGATG & chr17:63827498- & $y$ & $y$ & 124 & 1 \\
\hline 10 & PSMCS & $\mathrm{R}$ & СTCCGGAGGTTTTGGCTCTT & $\underline{\underline{63829528}}$ & $v$ & $v$ & 124 & 1 \\
\hline 11 & $\mathrm{MTCH}$ & $\mathrm{F}$ & CTTGGCGTAGGTGAAGAAGC & chr6:36978607- & 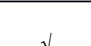 & $\sqrt{1}$ & 123 & 3 \\
\hline 11 & Мा & $R$ & СATCCCCTGCTCTACGTGAA & 36985885 & $v$ & $v$ & 123 & 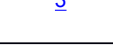 \\
\hline 12 & $M K R N 1$ & $\mathrm{~F}$ & GCAGCAAGGGATGACTTTGT & chr7:140459862- & - & $y$ & 98 & 3 \\
\hline 12 & $M K R N 1$ & $R$ & TGTATTTATGGAGACCGCTGC & $\underline{140471905}$ & - & $v$ & 98 & $\underline{3}$ \\
\hline & & $\mathrm{F}$ & TCTCATGCTGCTACTGTTCCT & chr20:35542342- & 1 & 1 & 159 & \\
\hline 13 & ERGIC3 & $\mathrm{R}$ & CAATACTCAGATAGGCACAAGGC & $\underline{35542833}$ & $v$ & $v$ & 152 & $\underline{4}$ \\
\hline 14 & $A D R M 1$ & $\mathrm{~F}$ & СAATGCTCCTCATCCTGGTC & chr20:62304519- & & $\sqrt{1}$ & 91 & 3 \\
\hline 14 & ADRMIII & $\mathrm{R}$ & GGAGGGTCTACGTGCTGAAG & $\underline{\underline{62306228}}$ & - & $v$ & 91 & $\underline{3}$ \\
\hline 15 & $R P 137 A$ & $\mathrm{~F}$ & TTCTGATGGCGGACTTTACC & chr2:216499955- & & $\sqrt{ }$ & 115 & 3 \\
\hline 15 & $R P L 3 / A$ & $\mathrm{R}$ & ATGAAGAGACGAGCTGTGGG & $\underline{\underline{216501378}}$ & - & $v$ & 115 & $\underline{3}$ \\
\hline 16 & $R P I 5$ & $\mathrm{~F}$ & TCGTATAGCAGCATGAGCTTTC & chr1:92837537- & 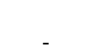 & $y$ & 136 & 3 \\
\hline 10 & RFLO & $\mathrm{R}$ & TGTTGCAGATTACATGCGCT & $\underline{92840589}$ & - & $v$ & 150 & 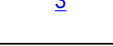 \\
\hline 17 & TSPAN31 & $\mathrm{F}$ & GGCTATTAACCGAAGCAAACAGA & chr12:57746235- & -4 & $\sqrt{ }$ & 117 & 4 \\
\hline It & TOFATVOT & $\mathrm{R}$ & GTGAGGTTGAATAAGCCACAACA & $\underline{57746683}$ & - & $v$ & 111 & $\underline{4}$ \\
\hline 18 & $D A R S$ & $\mathrm{~F}$ & AGCCGCAAGAGTCAGGAGA & chr2:135979349- & - & $\sqrt{ }$ & 124 & 4 \\
\hline 10 & ערוu & $\mathrm{R}$ & CCCGAACCAAAACTCGATCTG & $\underline{135985450}$ & -5 & r & 124 & $\underline{4}$ \\
\hline
\end{tabular}




\begin{tabular}{|c|c|c|c|c|c|c|c|c|c|c|c|}
\hline \multirow[b]{4}{*}{ Rank } & \multicolumn{11}{|c|}{ B } \\
\hline & \multicolumn{11}{|c|}{ Ranking of Candidate reference genes } \\
\hline & \multicolumn{2}{|c|}{ Genorm } & \multicolumn{2}{|c|}{ Normfinder } & \multicolumn{3}{|c|}{ Comparative $\mathrm{Ct}$} & \multicolumn{2}{|c|}{ Bestkeeper } & \multicolumn{2}{|c|}{ Reffinder } \\
\hline & Genes & $\begin{array}{c}\text { Stability } \\
\text { value (M) }\end{array}$ & Genes & $\begin{array}{c}\text { Stability } \\
\text { value (M) }\end{array}$ & Genes & $2^{\Lambda-(d e l C t)}$ & $\begin{array}{l}\text { Mean } \\
\text { Std.Dev }\end{array}$ & Genes & Std.dev (+-CP) & Genes & $\begin{array}{c}\text { Geomean } \\
\text { ranking }\end{array}$ \\
\hline 1 & RPL30 & 0.254 & PSMC5 & 0.004 & RPL27 & 1.13 & 0.34 & RPL27 & 0.278 & RPL30 & 1.32 \\
\hline 2 & RPL27 & 0.254 & MTCH1 & 0.005 & RPS13 & 0.90 & 0.43 & $R P L 30$ & 0.321 & $R P L 27$ & 1.57 \\
\hline 3 & PSMC5 & 0.348 & TSPAN21 & 0.006 & RPL30 & 1.21 & 0.46 & RPL5 & 0.362 & PSMC5 & 3.35 \\
\hline 4 & OAZ1 & 0.365 & DARS & 0.006 & RPL5 & 0.82 & 0.34 & RPS13 & 0.391 & $O A Z 1$ & 5.03 \\
\hline 5 & MKRN1 & 0.375 & RPL30 & 0.007 & RPL37A & 0.76 & 0.19 & MTCH1 & 0.419 & MTCH1 & 5.38 \\
\hline
\end{tabular}

4

5 Table 1: Primers used in the current study (A). The Reference column number

6 indicates- 1: primers designed for the study; 2: primers from Hendrik de Jonge et al.,

7 2007; 3: primers from Primerdepot (https://primerdepot.nci.nih.gov/); 4: primers from

8 primer bank (https://pga.mgh.harvard.edu/primerbank/); 5: primers from Campos et al,

9 2009. Results from different tools on the internal control genes and their ranks as

10 obtained using the validation samples (B). 